\title{
Avaliação do ponto de congelamento do leite cru por espectroscopia de infravermelho com transformada de Fourier como método de triagem
}

[Fourier Transform Infrared spectroscopy (FTIR) as a screening method for freezing point in raw milk]

E. Duarte ${ }^{1}$, J. Lima ${ }^{1}$, R.P. Fonseca ${ }^{2}$, M.O. Leite ${ }^{1}$, L.M. Fonseca ${ }^{\text {* }}$

${ }^{1}$ Escola de Veterinária - Universidade Federal de Minas Gerais - Belo Horizonte, MG

${ }^{2}$ Instituto de Ciências Exatas - Universidade Federal de Minas Gerais - Belo Horizonte, MG

\section{RESUMO}

O objetivo deste estudo foi investigar a interferência do teor de gordura do leite no ponto de congelamento (PC) obtido pelo infravermelho com transformada de Fourier (FTIR). Uma fração de leite cru foi desnatada, obtendo-se creme e leite desnatado. $\mathrm{O}$ leite integral e o desnatado foram adicionados com água $(0 \%, 2 \%$, $5 \%, 10 \%, 15 \%$ e $20 \%)$. O leite desnatado remanescente foi adicionado com creme $(5 \%, 10 \%, 15 \%, 20 \%)$ e água $(0 \%, 2 \%, 5 \%, 10 \%, 15 \%$ e $20 \%)$. As amostras foram analisadas para PC (crioscópio eletrônico e FTIR) e composição (FTIR). O PC medido por FTIR foi altamente correlacionado com o método do crioscópio eletrônico (acima de 98,5\%) e, como esperado, a adição de água foi significativa $(\mathrm{P} \leq 0,001)$ ao aumentar o PC do leite em ambos os métodos. No entanto, o alto teor de gordura no leite cru resultou na redução do PC ao se utilizar o FTIR, enquanto o baixo teor de gordura resultou em aumento do PC $(\mathrm{P} \leq 0,001)$. Uma vez que a adição de água causou o efeito inverso do alto teor de gordura no $\mathrm{PC}$ medido por FTIR, é importante considerar o teor de gordura do leite para evitar interpretações erradas do PC quando se utiliza o método FTIR.

Palavras-chave: ponto de congelamento, infravermelho com transformada de Fourier (FTIR), composição

\begin{abstract}
The objective of this study was to investigate the interference of milk fat content upon the Freezing Point (FP) obtained by Fourier-transform infrared (FTIR). A fraction of raw milk was skimmed, obtaining cream and skim milk. Whole and skim milk were added with water (0, 2, 5, 10, 15, and 20\%). The remaining skim milk was added with cream $(5,10,15,20 \%)$ and water (0, 2, 5, 10, 15, and 20\%). Samples were analyzed for FP (thermistor cryoscope and FTIR) and composition (FTIR). FP measured by FTIR was highly correlated with the thermistor cryoscope method (above 98.5\%) and, as expected, water addition was significant $(P \leq 0.001)$ upon increasing milk FP in both methods. However, high fat content in raw milk resulted in decreasing FP when using FTIR, while low fat content resulted in increased FP $(P \leq 0.001)$. Since water addition caused the inverse effect of high fat on FP measured by FTIR, it is important to regard the fat content of milk to avoid misinterpretation of FP when using the FTIR method.
\end{abstract}

Keywords: freezing point, fourier transform infrared (FTIR), composition

\section{INTRODUÇÃO}

O ponto de congelamento (PC) é considerado um importante indicador da qualidade do leite, utilizado principalmente para verificar adulteração pela adição de água. É uma análise empregada praticamente em todo o mundo por ser considerada uma técnica oficial precisa e pelo fato de os resultados apresentarem pouca variabilidade (Shipe, 1959; Matysek et al., 2011; Zagorska e Ciprovica, 2013).

Recebido em 31 de janeiro de 2019

Aceito em 20 de setembro de 2019

Autor para correspondência (corresponding author)

E-mail: leorges@gmail.com 
A metodologia de referência utilizada para determinar o PC do leite é por meio de crioscópios eletrônicos (Milk..., 2009). No entanto, esse método apresenta algumas desvantagens por ser laborioso, gerando grande demanda de tempo para realizar análise de um único parâmetro. Por isso, muitos laboratórios de análise da qualidade do leite fazem uso de equipamentos eletrônicos, como os baseados na espectroscopia de infravermelho com transformada de Fourier (FTIR - Fourier Transform Infrared), que possibilitam análise de multiparâmetros e de um grande número de amostras, demonstrando ser um método rápido e preciso (Rodriguez-Otero et al., 1997; Silveira et al., 2004; Stuart, 2004; Rodriguez-Saona et al., 2006; Sánchez et al., 2007).

O PC é uma medida diretamente ligada aos componentes solúveis do leite, em especial a lactose e os cloretos, que representam $80 \%$ do total do PC. O restante $(20 \%)$ são outros constituintes dissolvidos na água do leite, tais como ácido lático, fosfatos, citratos, potássio, cálcio, magnésio (Brathen, 1983; Zagorska e Ciprovica, 2013; Fagnani et al., 2014). Considerase que a proteína e a gordura não interferem no PC do leite, pois estão em solução coloidal e na forma de emulsão, respectivamente. Porém, alguns estudos verificaram que as proteínas podem promover variação no $\mathrm{PC}$ de forma indireta (Henno et al., 2008; Sala et al., 2010). Já em relação à gordura, existem poucos estudos com o objetivo de verificar sua influência sobre o PC determinado por metodologia de referência ou por espectroscopia de infravermelho com transformada de Fourier (Gencurova et al., 2008; Senevirathne et al., 2016).

Assim, o objetivo deste trabalho foi verificar a interferência de diferentes teores de gordura e adição de água ao leite na crioscopia avaliada por espectroscopia de infravermelho com transformada de Fourier e comparar os resultados com o método de referência utilizando-se crioscópio eletrônico.

\section{MATERIAL E MÉTODOS}

As amostras de leite utilizadas para o experimento consistiram de leite cru de conjunto, coletadas em tanque refrigerador, proveniente de vacas da Fazenda Experimental da Escola de Veterinária da Universidade Federal de Minas Gerais, localizada no município de Igarapé, Minas Gerais, Brasil. O experimento foi realizado em cinco repetições; em cada uma foram utilizados 15 litros de leite cru integral. O leite foi homogeneizado por agitação, transferido para recipientes previamente limpos e sanitizados, transportado e armazenado em câmara fria sob refrigeração $\left(4^{\circ} \mathrm{C}\right)$. Foi realizada análise de acidez titulável (Official..., 2016) para se verificar o estado de conservação do leite. Em seguida, foi feito o preparo das amostras do experimento 1 .

Aproximadamente $95 \%$ do volume total do leite cru integral foram aquecidos até $40^{\circ} \mathrm{C}$ e submetidos à centrifugação mecânica (desnatadeira) para separação da fase gordurosa, obtendo-se leite desnatado e creme. Uma porção do leite desnatado e uma parcela dos $5 \%$ restantes do leite cru integral foram adicionadas de diferentes porcentagens de água $(0 \%, 2 \%, 5 \%$, $10 \%, 15 \%$ e $20 \%$ ) em 12 frascos no total, com capacidade de $40 \mathrm{~mL}$ e com conservante bronopol (2-bromo-2-nitro-1,3-propanediol) (experimento $1)$.

$\mathrm{O}$ experimento 2 foi realizado com a outra parte do leite desnatado, que foi subdividido $\mathrm{e}$ transferido para 24 frascos de $40 \mathrm{~mL}$ com conservante bronopol, adicionados de diferentes porcentagens de creme $(5 \%, 10 \%, 15 \%$ e $20 \%$ de creme, que correspondeu, em média, a 2,5\%, 5\%, $7,4 \%$ e $9,9 \%$ de gordura, respectivamente) e de água $(0 \%, 2 \%, 5 \%, 10 \%, 15 \%$ e $20 \%)$. As amostras foram homogeneizadas por inversão, por cinco vezes, e armazenadas, de um dia para o outro, em câmara fria, com temperatura de $4^{\circ} \mathrm{C}$. No dia seguinte, as amostras foram homogeneizadas por inversão, por cinco vezes, e analisadas quanto ao PC pela metodologia de referência (Crioscópio Eletrônico LK - 7000; Laktron, Londrina, PR).

Em seguida, as réplicas das mesmas amostras foram pré-aquecidas por exatamente 20 minutos, em banho-maria, a uma temperatura de $40 \pm 2^{\circ} \mathrm{C}$, homogeneizadas por inversão, por cinco vezes, e analisadas quanto ao PC e à composição (teores de gordura, proteína, lactose, sólidos totais) (CombiScope $^{\mathrm{TM}}$ FTIR; Delta Instruments, Drachten, Holanda). O Crioscópio Eletrônico LK - 7000 (Laktron, Londrina, PR) foi calibrado com soluções-padrão de cloreto de sódio $\left(-0,422^{\circ} \mathrm{H}\right.$; $-0,530^{\circ} \mathrm{H}$ e $\left.-0,621^{\circ} \mathrm{H}\right)($ Milk..., 2009). 
A calibração do equipamento CombiScope FTIR foi realizada por meio de 14 amostras- padrão de leite cru com diferentes faixas de composição para gordura, proteína, lactose e extrato seco total. A calibração foi feita utilizando-se o programa de calibração do próprio equipamento. As amostraspadrão foram adquiridas do Laboratório Valacta (Dairy Production Centre of Expertise, Quebec, Canadá). A calibração para leitura da crioscopia foi feita com amostras-padrão fornecidas pelo laboratório DQCI SERVICES (Dairy Quality Control Incorporated, Estados Unidos). As amostras-padrão foram fornecidas juntamente com os laudos contendo os resultados das análises de referência realizadas no laboratório de origem. As análises foram realizadas no Laboratório de Análise da Qualidade do Leite da Escola de Veterinária da UFMG (Acreditado ABNT NBR ISO/IEC 17025 e credenciado pelo Ministério da Agricultura, Pecuária e Abastecimento).

Foi realizada análise de regressão linear múltipla, com significância de 95\%, para os dois experimentos, aplicada por meio do pacote estatístico Stata 12.0 (Stata Corp., versão 12). A variável dependente foi o $\mathrm{PC}$, e as variáveis independentes no experimento 1 foram tipo de leite, porcentagem de água e metodologia e, no experimento 2, foram porcentagem de gordura, porcentagem de água e metodologia. As equações de regressão linear múltipla para os experimentos foram:

experimento 1:

$$
P C=\beta_{0}+\beta_{1} A+\beta_{2} L+\beta_{3} M+\beta_{4} L M+\varepsilon
$$

$\mathrm{PC}=$ ponto de congelamento;

$\beta_{0}=$ constante;

$\beta_{1}=$ coeficiente de regressão dos teores de água (A) que vão de $0 \%$ a $20 \%$;

$\beta_{2}=$ coeficiente de regressão do tipo de leite (L), em que 0 (zero) é leite desnatado e 1 (um) é leite cru integral;

$\beta_{3}=$ coeficiente de regressão da metodologia (M), em que 0 (zero) é a metodologia de referência (crioscópio eletrônico) e 1 (um) é a metodologia de espectroscopia de FTIR; $\beta_{4}=$ coeficiente de regressão da interação entre tipo de leite (L) e metodologia (M);

$\varepsilon=$ erro;

experimento 2 :

$P C=\beta_{0}+\beta_{1} A+\beta_{2} G+\beta_{3} M+\beta_{4} G M+\varepsilon$

$\mathrm{PC}=$ ponto de congelamento;

$\beta_{0}=$ constante;

$\beta_{1}=$ coeficiente de regressão dos teores de água (A) que vão de $0 \%$ a $20 \%$;

$\beta_{2}=$ coeficiente de regressão dos teores de gordura (G) que vão, em média, de $2,5 \%$ a $9,9 \%$;

$\beta_{3}=$ coeficiente de regressão da metodologia (M), em que 0 (zero) é a metodologia de referência (crioscópio eletrônico) e 1 (um) é a metodologia de espectroscopia de FTIR;

$\beta_{4}=$ coeficiente de regressão da interação entre teores de gordura $(\mathrm{G})$ e metodologia $(\mathrm{M})$;

$\varepsilon=$ erro.

Foram calculados fatores de correção para eliminação do efeito da adição do conservante bronopol nos resultados do PC pela metodologia de referência e espectroscopia de FTIR. Para isso, foi utilizada comparação de pares (teste $\mathrm{t}$ ) e foram calculados os intervalos de confiança com significância de 95\% (Sampaio, 2015), utilizandose o programa estatístico Stata 12.0 (Stata Corp., versão 12).

\section{RESULTADOS E DISCUSSÃO}

O resultado médio da acidez titulável do leite foi de $15,2^{\circ} \mathrm{D} \pm 0,59$ (graus Dornic), o que demonstra que o leite estava em bom estado de conservação. O resultado encontrado está de acordo com a legislação brasileira vigente (Brasil, 2018). Na Tab. 1, estão apresentados os intervalos de confiança e os fatores de correção para corrigir os efeitos da adição do conservante bronolat sobre o PC do leite nas duas metodologias. $\mathrm{O}$ fator de correção para o crioscópio eletrônico foi de $0,006^{\circ} \mathrm{C}$, enquanto para o FTIR, o fator de correção foi de $0,005^{\circ} \mathrm{C}$.

Tabela 1. Fator de correção e intervalo de confiança do PC obtido por crioscópio eletrônico e o FTIR

\begin{tabular}{llll} 
Metodologia analítica & $\mathrm{N}$ & Média ${ }^{1} \mathrm{PC}\left({ }^{\circ} \mathrm{C}\right)$ & $\mathrm{IC}^{2}(95 \%)$ \\
\hline Crioscópio eletrônico $^{3}$ & 15 & 0,006 & $0,005 \mathrm{H} 0,007$ \\
FTIR $^{4}$ & 15 & 0,005 & $0,004-10,006$ \\
\hline
\end{tabular}

${ }^{1}$ Média da diferença calculada a partir dos valores de PC com e sem adição de conservante bronolat. ${ }^{2}$ Intervalo de confiança em nível de 95\%. ${ }^{3}$ Crioscópio eletrônico (LK - 7000, Laktron). ${ }^{4}$ Espectroscopia de infravermelho com transformada de Fourier (LactoScope ${ }^{\mathrm{TM}}$ FTIR). 
Como esperado, os componentes do leite foram diluídos proporcionalmente após a adição de água com consequente perda nutricional (Tab. 2). Os teores de gordura, proteína e sólidos totais foram considerados normais, de acordo com a legislação brasileira, até $10 \%$ de adição de água, o que ressalta a importância da análise complementar, como o PC para triagem de amostras adulteradas. O PC do leite está correlacionado com os componentes solúveis, principalmente lactose e cloretos. Portanto, quando há adição de água, mesmo em pequenas quantidades, o PC se aproxima de $0^{\circ} \mathrm{C}$ (Zagorska e Ciprovica, 2013), como pode ser observado na Tab. 3 .

Os dados da Tab. 3 foram plotados na Fig. 1, na qual é possível notar linearidade nos dois métodos e, embora ambas as curvas não se sobreponham, a diferença é menor para o leite integral.

Tabela 2. Composição do leite cru (desnatado e integral) obtido por espectroscopia de infravermelho com transformada de Fourier (FTIR) após a adição de água (média +desvio-padrão)

\begin{tabular}{cccccc}
\hline Água $(\%)$ & Leite & $\begin{array}{c}\text { Gordura } \\
(\mathrm{g} / 100 \mathrm{~g})\end{array}$ & $\begin{array}{c}\text { Lactose } \\
(\mathrm{g} / 100 \mathrm{~g})\end{array}$ & $\begin{array}{c}\text { Proteína } \\
(\mathrm{g} / 100 \mathrm{~g})\end{array}$ & $\begin{array}{c}\text { Sólidos totais } \\
(\mathrm{g} / 100 \mathrm{~g})\end{array}$ \\
\hline \multirow{2}{*}{0} & Desnatado & $0,23 \pm 0,01$ & $4,91 \pm 0,036$ & $3,35 \pm 0,057$ & $9,38 \pm 0,08$ \\
& Integral & $4,09 \pm 0,73$ & $4,69 \pm 0,036$ & $3,27 \pm 0,061$ & $12,79 \pm 0,79$ \\
2 & Desnatado & $0,22 \pm 0,01$ & $4,79 \pm 0,033$ & $3,29 \pm 0,059$ & $9,20 \pm 0,09$ \\
& Integral & $3,88 \pm 0,68$ & $4,59 \pm 0,022$ & $3,20 \pm 0,054$ & $12,65 \pm 0,68$ \\
\multirow{2}{*}{5} & Desnatado & $0,22 \pm 0,01$ & $4,64 \pm 0,022$ & $3,19 \pm 0,062$ & $8,94 \pm 0,08$ \\
& Integral & $3,74 \pm 0,67$ & $4,44 \pm 0,028$ & $3,11 \pm 0,054$ & $12,27 \pm 0,66$ \\
\multirow{2}{*}{10} & Desnatado & $0,21 \pm 0,01$ & $4,38 \pm 0,022$ & $3,03 \pm 0,056$ & $8,52 \pm 0,08$ \\
& Integral & $3,61 \pm 0,61$ & $4,19 \pm 0,022$ & $2,95 \pm 0,052$ & $11,75 \pm 0,62$ \\
\multirow{2}{*}{15} & Desnatado & $0,21 \pm 0,01$ & $4,12 \pm 0,015$ & $2,87 \pm 0,056$ & $8,10 \pm 0,08$ \\
& Integral & $3,41 \pm 0,58$ & $3,94 \pm 0,026$ & $2,80 \pm 0,050$ & $11,15 \pm 0,59$ \\
& Desnatado & $0,20 \pm 0,01$ & $3,85 \pm 0,024$ & $2,71 \pm 0,048$ & $7,69 \pm 0,08$ \\
& Integral & $3,22 \pm 0,54$ & $3,69 \pm 0,027$ & $2,64 \pm 0,049$ & $10,55 \pm 0,54$ \\
\hline
\end{tabular}

Tabela 3. Ponto de congelamento do leite cru (leite integral e desnatado) obtido pelo crioscópio eletrônico e por espectroscopia de infravermelho com transformada de Fourier (FTIR) após a adição de água (média \pm desvio-padrão)

\begin{tabular}{clll}
\hline$\%$ Água & Leite & $\begin{array}{l}\mathrm{PC}\left({ }^{\circ} \mathrm{C}\right) \\
\text { Criosc. eletr. }\end{array}$ & $\begin{array}{l}\text { PC }\left({ }^{\circ} \mathrm{C}\right) \\
\text { FTIR }^{2}\end{array}$ \\
\hline \multirow{2}{*}{0} & $\begin{array}{l}\text { Desnatado } \\
\text { Integral }\end{array}$ & $-0,516 \pm 0,004$ & $-0,495 \pm 0,003$ \\
\multirow{2}{*}{2} & Desnatado & $-0,515 \pm 0,003$ & $-0,526 \pm 0,009$ \\
& Integral & $-0,504 \pm 0,003$ & $-0,484 \pm 0,003$ \\
\multirow{2}{*}{5} & Desnatado & $-0,487 \pm 0,002$ & $-0,517 \pm 0,009$ \\
& Integral & $-0,487 \pm 0,003$ & $-0,468 \pm 0,003$ \\
\multirow{2}{*}{10} & Desnatado & $-0,460 \pm 0,003$ & $-0,500 \pm 0,008$ \\
& Integral & $-0,460 \pm 0,003$ & $-0,442 \pm 0,002$ \\
\multirow{2}{*}{15} & Desnatado & $-0,434 \pm 0,003$ & $-0,474 \pm 0,007$ \\
& Integral & $-0,432 \pm 0,003$ & $-0,416 \pm 0,002$ \\
\multirow{2}{*}{20} & Desnatado & $-0,407 \pm 0,003$ & $-0,446 \pm 0,007$ \\
& Integral & $-0,403 \pm 0,004$ & $-0,390 \pm 0,002$ \\
\hline
\end{tabular}

${ }^{1}$ Crioscópio eletrônico (LK - 7000, Laktron). ${ }^{2}$ Espectroscopia de infravermelho com transformada de Fourier (LactoScope ${ }^{\text {TM }}$ FTIR). 
(A)

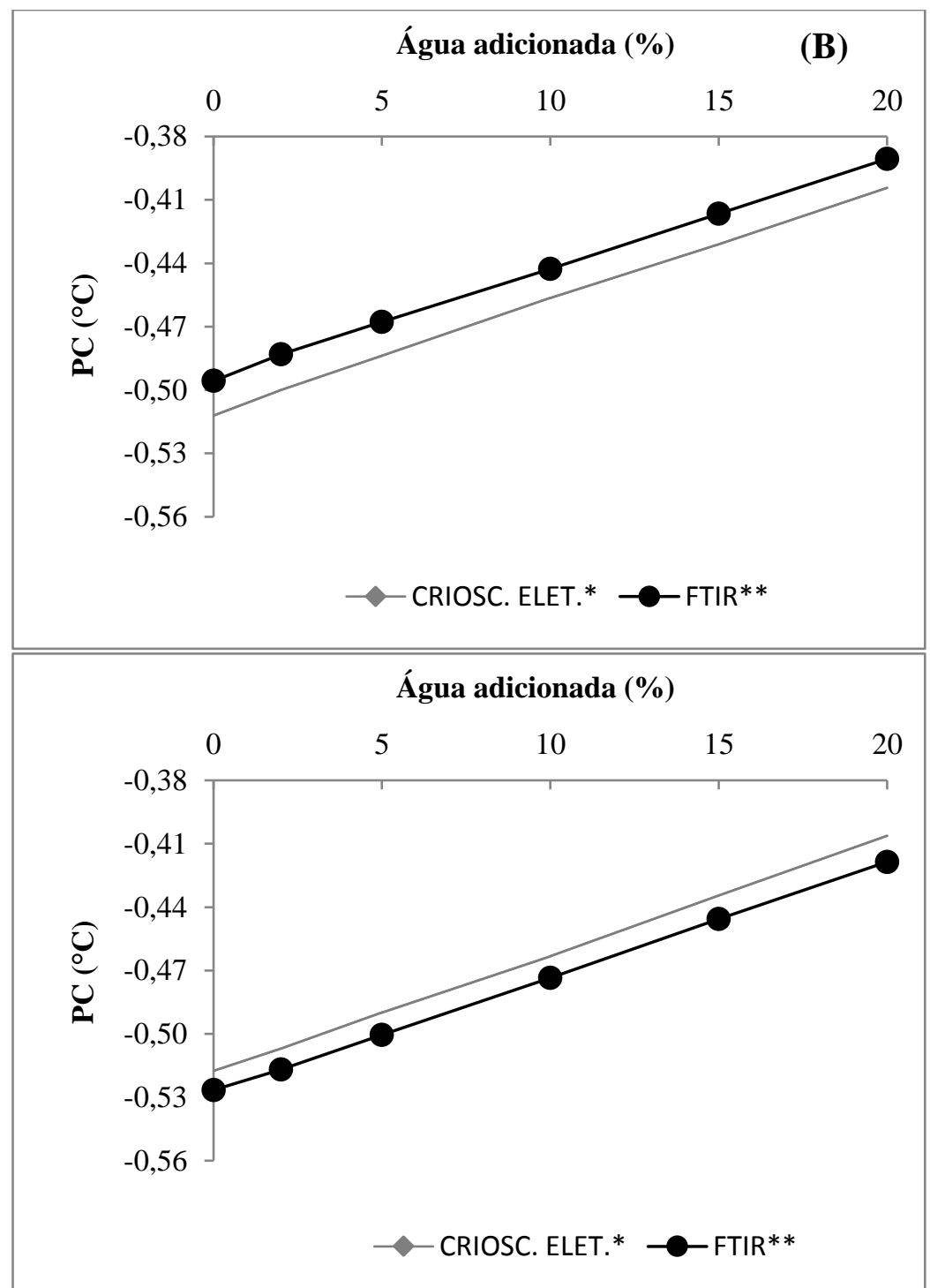

* Crioscópio eletrônico (LK - 7000, Laktron). **Espectroscopia de infravermelho com transformada de Fourier (LactoScope ${ }^{\mathrm{TM}}$ FTIR).

Figura 1. Linearidade do PC do leite desnatado (A) e do leite integral (B) adicionados de água e avaliados por crioscopia eletrônica e espectroscopia de FTIR.

Para o leite integral, o PC obtido por meio do FTIR foi mais próximo do PC medido com o crioscópio eletrônico. No entanto, para o leite desnatado, os resultados foram invertidos, com maiores valores do PC com o método FTIR.

O coeficiente de determinação para o experimento 1 foi alto $(98,7 \%)$, mostrando um ótimo ajuste entre o PC e as variáveis avaliadas (Tab. 4). Em média, o PC obtido pelo FTIR aumentou $0,019^{\circ} \mathrm{C}$ $(\mathrm{P}<0,001)$ quando comparado ao método de referência (crioscópio eletrônico). Essa diferença foi mais evidente para o leite desnatado, independentemente da quantidade de adição de água (Tab. 4).

O teor de gordura do leite (desnatado ou integral) não afetou o PC medido pelo método de referência. No entanto, o método analítico foi um fator significativo $(\mathrm{P}<0,001)$. O PC do leite integral com diferentes porcentagens de água analisadas pelo FTIR foi capaz de diminuir - 
$0,032^{\circ} \mathrm{C} \quad(\mathrm{P}<0,001)$. Para cada $1 \%$ de água adicionada, o PC aumenta $0,0054^{\circ} \mathrm{C}$. Resultados semelhantes foram verificados por Sánchez et al. (2007), que encontraram aumento do PC de $0,006^{\circ} \mathrm{C}$ para cada $1 \%$ de água adicionada ao leite de cabra (tratamento com adição de $0 \%, 1 \%, 2 \%$,
$3 \%, 4 \%, 5 \%, 6 \%$ e 7\%). Hanus et al. (2012) encontraram aumento do $\mathrm{PC}$ igual a $0,006^{\circ} \mathrm{C}$ para cada $1 \%$ de água adicionada no leite de vaca, porém a faixa de adição foi de até $2,5 \%$ de água $(0 \%, 0,5 \%, 1 \%, 1,5 \%, 2 \%$ e $2,5 \%)$.

Tabela 4. Análise do modelo de regressão linear múltipla para variável do ponto de congelamento do leite e adição de água, leite (desnatado ou leite integral) e método analítico (crioscópio eletrônico ou FTIR)

\begin{tabular}{ccccc}
\hline Variáveis & Coeficientes & Valor-P & $\mathrm{PC}(95 \%)$ & $\mathrm{R}^{2}$ ajustado $(\%)$ \\
\hline Constante & $-0,512$ & $<0,001$ & $-0,515 \mathrm{H}-0,510$ & \\
Água & 0,0054 & $<0,001$ & $0,0053 \mathrm{H} 0,0055$ & \\
Leite & 0,001 & 0,411 & $-0,001 \mathrm{H}-0,003$ & 98,66 \\
Metodologia & 0,019 & $<0,001$ & $0,016 \mathrm{H}-0,021$ & \\
Leite x Metodologia & $-0,032$ & $<0,001$ & $-0,035 \mathrm{H}-0,029$ & \\
\hline
\end{tabular}

Para o experimento 2, leite desnatado com gordura (creme) e água, os resultados obtidos por FTIR para lactose e proteína diminuíram por terem sido afetados pela adição de água e creme, enquanto os sólidos totais aumentaram proporcionalmente ao teor de gordura (Tab. 5).

Tabela 5. Composição do leite desnatado adicionado de água e de creme e analisado por espectroscopia de infravermelho com transformada de Fourier (FTIR) (média \pm desvio-padrão)

\begin{tabular}{ccccc}
\hline Água $(\%)$ & $\begin{array}{c}\text { Gordura } \\
(\mathrm{g} / 100 \mathrm{~g})\end{array}$ & $\begin{array}{c}\text { Lactose } \\
(\mathrm{g} / 100 \mathrm{~g})\end{array}$ & $\begin{array}{c}\text { Proteína } \\
(\mathrm{g} / 100 \mathrm{~g})\end{array}$ & $\begin{array}{c}\text { Sólidos totais } \\
(\mathrm{g} / 100 \mathrm{~g})\end{array}$ \\
\hline \multirow{2}{*}{0} & 2,53 & $4,76 \pm 0,020$ & $3,30 \pm 0,058$ & $11,55 \pm 0,19$ \\
& 5,10 & $4,66 \pm 0,028$ & $3,22 \pm 0,060$ & $13,96 \pm 0,16$ \\
& 7,36 & $4,54 \pm 0,042$ & $3,13 \pm 0,056$ & $15,97 \pm 0,46$ \\
2 & 10 & $4,40 \pm 0,042$ & $3,03 \pm 0,052$ & $18,25 \pm 0,64$ \\
& 2,58 & $4,67 \pm 0,029$ & $3,24 \pm 0,058$ & $11,45 \pm 0,20$ \\
& 5,14 & $4,55 \pm 0,031$ & $3,16 \pm 0,055$ & $13,83 \pm 0,25$ \\
& 7,37 & $4,44 \pm 0,027$ & $3,07 \pm 0,057$ & $15,82 \pm 0,27$ \\
& 9,84 & $4,31 \pm 0,040$ & $2,97 \pm 0,046$ & $17,96 \pm 0,48$ \\
& 2,59 & $4,51 \pm 0,031$ & $3,14 \pm 0,060$ & $11,21 \pm 0,24$ \\
& 5,12 & $4,40 \pm 0,028$ & $3,07 \pm 0,053$ & $13,57 \pm 0,22$ \\
& 7,56 & $4,28 \pm 0,027$ & $2,96 \pm 0,050$ & $15,74 \pm 0,26$ \\
& 9,98 & $4,14 \pm 0,033$ & $2,87 \pm 0,042$ & $17,82 \pm 0,53$ \\
& 2,60 & $4,25 \pm 0,022$ & $2,98 \pm 0,061$ & $10,82 \pm 0,20$ \\
& 5,02 & $4,14 \pm 0,031$ & $2,91 \pm 0,055$ & $13,07 \pm 0,35$ \\
& 7,45 & $4,01 \pm 0,021$ & $2,81 \pm 0,049$ & $15,22 \pm 0,35$ \\
& 9,83 & $3,88 \pm 0,039$ & $2,71 \pm 0,041$ & $17,27 \pm 0,60$ \\
& 2,49 & $3,99 \pm 0,021$ & $2,82 \pm 0,053$ & $10,29 \pm 0,18$ \\
& 5,07 & $3,88 \pm 0,031$ & $2,75 \pm 0,053$ & $12,70 \pm 0,24$ \\
& 7,41 & $3,75 \pm 0,019$ & $2,65 \pm 0,050$ & $14,77 \pm 0,31$ \\
& 9,93 & $3,55 \pm 0,107$ & $2,52 \pm 0,066$ & $16,85 \pm 0,67$ \\
& 2,55 & $3,73 \pm 0,016$ & $2,65 \pm 0,050$ & $9,94 \pm 0,21$ \\
& 5,10 & $3,61 \pm 0,017$ & $2,58 \pm 0,047$ & $12,30 \pm 0,22$ \\
& 7,36 & $3,49 \pm 0,023$ & $2,49 \pm 0,041$ & $14,30 \pm 0,33$ \\
& 9,93 & $3,40 \pm 0,124$ & $2,43 \pm 0,077$ & $16,60 \pm 0,66$ \\
\hline
\end{tabular}

A Tab. 6 apresenta os valores médios do PC obtidos pela metodologia de referência e por FTIR em diferentes condições analíticas testadas. Os valores encontrados por esse último método (- $0,582^{\circ} \mathrm{C}$ a $-0,413^{\circ} \mathrm{C}$ ) foram menores do que os obtidos pelo método de referência $\left(-0,516^{\circ} \mathrm{C}\right.$ a $0,395^{\circ} \mathrm{C}$ ) na maioria das condições analíticas estudadas. 
Tabela 6. Ponto de congelamento do leite após adição de água e creme (50\% de gordura), analisado por crioscópio eletrônico e FTIR (média \pm desvio-padrão)

\begin{tabular}{cccc}
\hline Água $(\%)$ & Gordura $(\%)$ & $\begin{array}{c}\text { PC }\left({ }^{\circ} \mathrm{C}\right) \\
\text { Criosc. eletr. }\end{array}$ & $\begin{array}{c}\text { PC }\left({ }^{\circ} \mathrm{C}\right) \\
\text { FTIR }^{2}\end{array}$ \\
\hline \multirow{2}{*}{0} & 2,53 & $-0,515 \pm 0,004$ & $-0,514 \pm 0,004$ \\
& 5,10 & $-0,516 \pm 0,004$ & $-0,541 \pm 0,003$ \\
& 7,36 & $-0,516 \pm 0,003$ & $-0,560 \pm 0,006$ \\
2 & 10 & $-0,516 \pm 0,003$ & $-0,582 \pm 0,009$ \\
& 2,58 & $-0,505 \pm 0,003$ & $-0,507 \pm 0,004$ \\
& 5,14 & $-0,504 \pm 0,004$ & $-0,531 \pm 0,004$ \\
& 7,37 & $-0,506 \pm 0,004$ & $-0,551 \pm 0,004$ \\
5 & 9,84 & $-0,505 \pm 0,003$ & $-0,571 \pm 0,006$ \\
& 2,59 & $-0,487 \pm 0,004$ & $-0,490 \pm 0,004$ \\
& 5,12 & $-0,487 \pm 0,004$ & $-0,516 \pm 0,003$ \\
& 7,56 & $-0,487 \pm 0,005$ & $-0,537 \pm 0,003$ \\
& 9,98 & $-0,486 \pm 0,004$ & $-0,556 \pm 0,007$ \\
& 2,60 & $-0,459 \pm 0,004$ & $-0,466 \pm 0,004$ \\
& 5,02 & $-0,459 \pm 0,004$ & $-0,489 \pm 0,004$ \\
& 7,45 & $-0,458 \pm 0,003$ & $-0,509 \pm 0,005$ \\
& 9,83 & $-0,455 \pm 0,002$ & $-0,528 \pm 0,008$ \\
& 2,49 & $-0,431 \pm 0,004$ & $-0,438 \pm 0,003$ \\
& 5,07 & $-0,431 \pm 0,004$ & $-0,463 \pm 0,003$ \\
& 7,41 & $-0,428 \pm 0,004$ & $-0,483 \pm 0,004$ \\
& 9,93 & $-0,425 \pm 0,003$ & $-0,497 \pm 0,013$ \\
& 2,55 & $-0,404 \pm 0,004$ & $-0,413 \pm 0,003$ \\
& 5,10 & $-0,402 \pm 0,003$ & $-0,437 \pm 0,003$ \\
& 7,36 & $-0,399 \pm 0,003$ & $-0,456 \pm 0,004$ \\
& 9,93 & $-0,395 \pm 0,003$ & $-0,482 \pm 0,012$ \\
\hline
\end{tabular}

Da mesma forma, para o experimento 2, os resultados de regressão linear múltipla mostram que os dados são ajustados (R2=98,95\%) (Tab. 7). Ambos os métodos, crioscópio eletrônico e FTIR, foram diferentes $(\mathrm{P}<0,001)$ quando a composição do leite foi modificada, com diferença média de $0,019^{\circ} \mathrm{C}$.

Tabela 7. Análise do modelo de regressão linear múltipla para a variável ponto de congelamento do leite e adição de água e/ou creme e método analítico (crioscópio eletrônico ou FTIR)

\begin{tabular}{ccccc}
\hline Variáveis & Coeficientes & Valor-P & IC $(95 \%)$ & $\mathrm{R}^{2}$ ajustado (\%) \\
\hline Constante & $-0,515$ & $<0,001$ & $-0,518$ - 0,513 & \\
Água & 0,0055 & $<0,001$ & $0,0054-0,0056$ & \\
Gordura & 0,0005 & 0,004 & $0,0001-0,0008$ & 98,93 \\
Metodologia & 0,019 & $<0,001$ & $0,016-0,022$ & \\
Gordura x Metodologia & $-0,0093$ & $<0,001$ & $-0,0100-0,0915$ & \\
\hline
\end{tabular}

Os resultados do presente estudo são semelhantes aos encontrados por Conrado et al. (2012), que não verificaram diferença significativa $(\mathrm{P}>0,05)$ entre o crioscópio eletrônico e o método FTIR, porém apenas para o leite com composição normal. No conhecimento dos autores do presente trabalho, o desvio do PC devido ao conteúdo anormal de gordura do leite não foi relatado anteriormente.
Embora, usando o método do crioscópio eletrônico, Sala et al. (2010) não tenham encontrado influência da gordura no PC do leite para amostras com teor de gordura de 1,7\% a $5,0 \%$, e Senevirathne et al. (2016) para amostras com três níveis de gordura $(<3 \%, 3 \%$ a $4 \%, 4 \%$ a $5 \%$, >5\%), o presente estudo encontrou uma pequena, mas significativa, diferença $\left(0,0005^{\circ} \mathrm{C}\right)$ na faixa de $2,5 \mathrm{~g} / 100 \mathrm{~mL}$ a $9,9 \mathrm{~g} / 100 \mathrm{~mL}(\mathrm{P}<0,05)$. Isso pode ser devido aos diferentes componentes 
solúveis da membrana dos glóbulos de gordura (El-Loly, 2011), uma vez que essas amostras foram obtidas por meio da adição de creme (50\% de gordura) ao leite. No entanto, a diferença do PC entre os métodos de referência (crioscópio eletrônico) e FTIR aumentou com o aumento do teor de gordura no leite $(\mathrm{P}<0,05)$, conforme observado na Fig. 2.

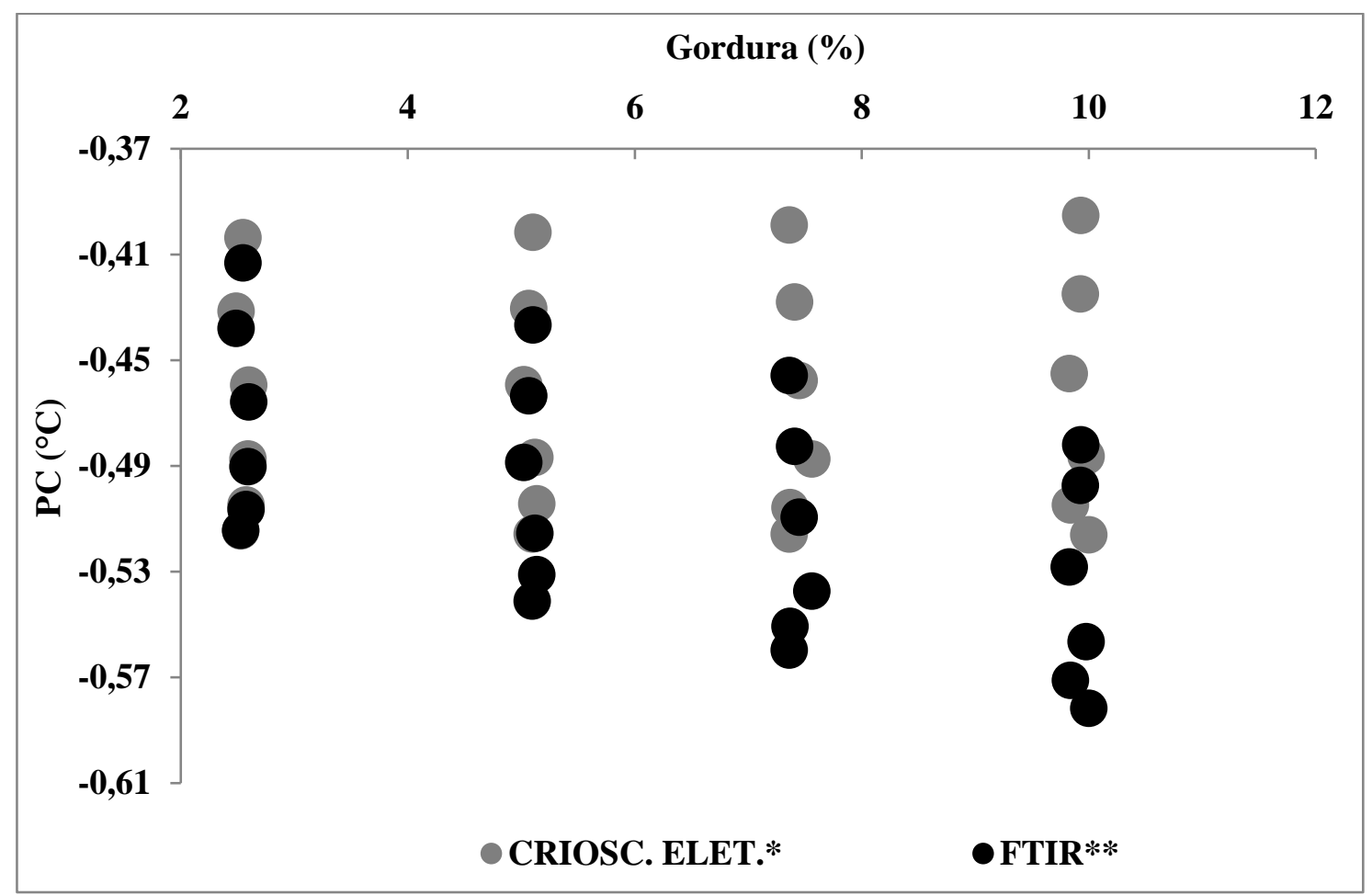

*Crioscópio eletrônico (LK - 7000, Laktron). **Espectroscopia de infravermelho com transformada de Fourier (LactoScope ${ }^{\mathrm{TM}}$ FTIR).

Figura 2. Ponto de congelamento (PC) do leite desnatado adicionado de gordura e água e analisado pelo método do crioscópio eletrônico e do FTIR.

A espectroscopia de FTIR é utilizada mundialmente para análise da qualidade do leite, com alto rendimento analítico e baixo custo, permitindo a determinação do PC como método de triagem (Pintic-Pukec et al., 2011). O presente estudo indica uma redução do $\mathrm{PC}$ de $0,0093^{\circ} \mathrm{C}$ para cada $1 \%$ de gordura e um aumento de $0,0055^{\circ} \mathrm{C}$ para cada $1 \%$ de água adicionada $(\mathrm{P}<0,001)$. Portanto, o efeito de adição de água no PC pode ser contrabalançado com o aumento do teor de gordura quando estimado pelo FTIR. Assim, é relevante levar em consideração o teor de gordura do leite ao se avaliar o PC estimado pelo FTIR (Fig. 3).

A influência do teor de gordura e da porcentagem de água sobre o PC do leite obtido pela metodologia de referência e por FTIR está apresentada na Fig. 3. Como pode ser observado, as duas técnicas são equivalentes quando não há alteração na composição do leite, ou seja, quando o teor de gordura está dentro dos padrões normais. No entanto, quando há alteração do teor de gordura do leite, o PC é alterado quando analisado por FTIR. 
(A)

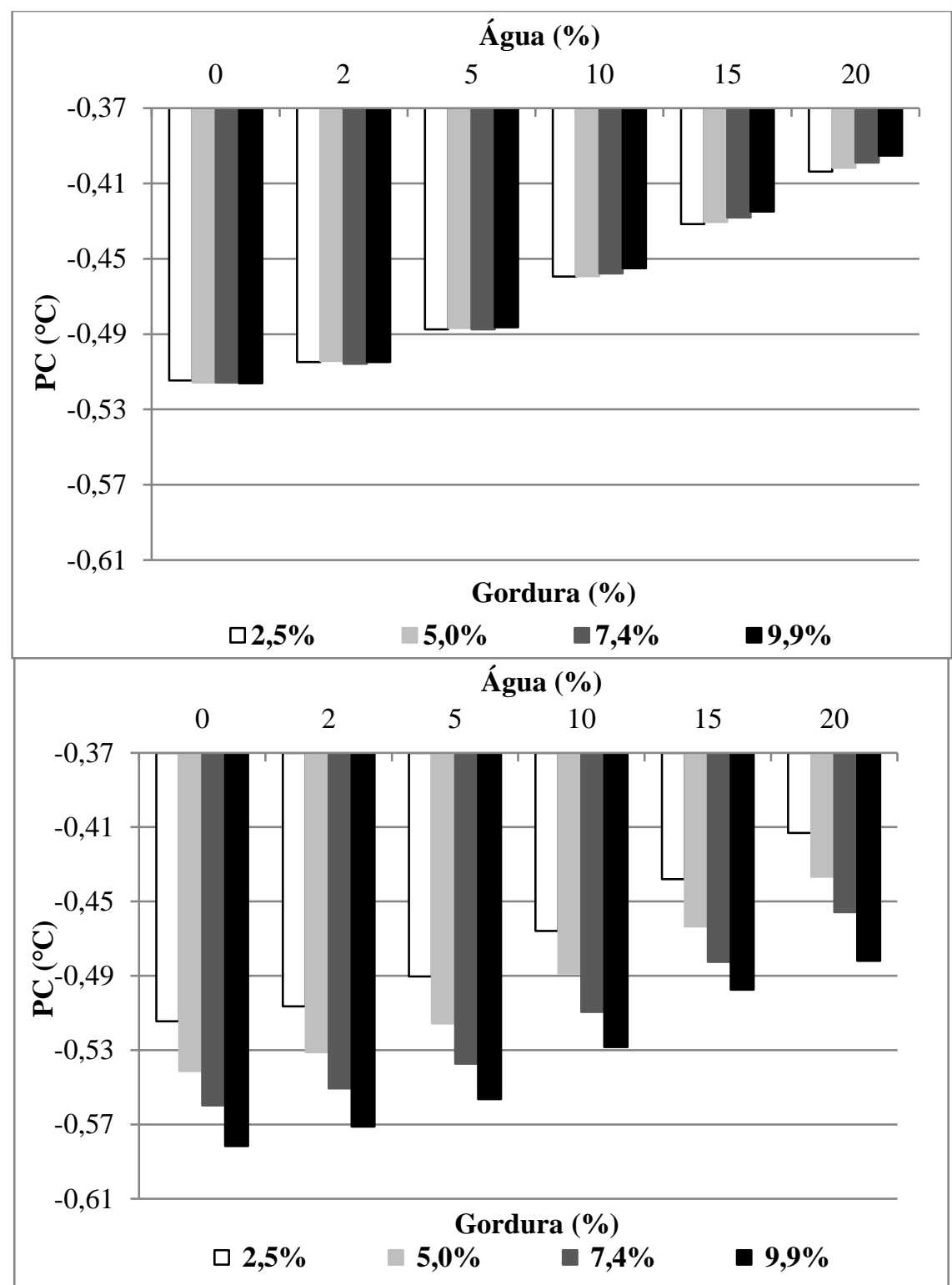

* Crioscópio eletrônico (LK - 7000, Laktron). ** Espectroscopia de infravermelho com transformada de Fourier (LactoScope ${ }^{\mathrm{TM}}$ FTIR).

Figura 3. PC do leite desnatado adicionado de diferentes porcentagens de água e gordura avaliado por crioscopia eletrônica* (A) e por espectroscopia de FTIR** (B).

\section{CONCLUSÃO}

A adição de água ao leite resultou em aumento do PC estimado pelo FTIR, enquanto o maior teor de gordura foi correlacionado com o menor PC e o menor teor de gordura com maior PC. A técnica FTIR é muito utilizada na rotina de análise nos laboratórios de análise da qualidade do leite cru. Portanto, a estimativa do PC do leite para amostras de leite com uma faixa anormal de conteúdo de gordura deve ser usada com cautela em razão da potencial má interpretação devido à interferência do conteúdo de gordura nesse método.

\section{AGRADECIMENTOS}

Ao apoio da Fundação de Apoio à Pesquisa de Minas Gerais (Fapemig) (CVZ-APQ-01179-14), do Conselho Nacional de Pesquisa (PQ-309801 / 2014-1), da Fundação de Desenvolvimento de 
Pesquisa (Fundep - Laboratório de Análise da Qualidade do Leite da EV-UFMG) e da bolsa de estudos concedida pela Coordenação de Aperfeiçoamento de Pessoal de Nível Superior (Capes).

\section{REFERÊNCIAS}

BRASIL. Ministério de Agricultura Pecuária e Abastecimento (MAPA). Instrução Normativa $n^{\circ} 76$, de 26 de novembro de 2018. Aprova os Regulamentos Técnicos que fixam a identidade e as características de qualidade que devem apresentar o leite cru refrigerado, o leite pasteurizado e o leite pasteurizado tipo A. Diário Oficial da União, Brasília, DF., 26 nov. 2018. Seção 1, n.230.

BRATHEN, G. Measurement of extraneous water by the freezing point test. Bull. Int. Dairy Fed., n.154, p.5, 1983.

CONRRADO, R.S.; OLIVEIRA, M.C.P.P.; FONSECA, L.M. et al. Freezing point of raw milk by Fourier transform infrared methodology (FTIR). J. Anim. Sci., v.90, Suppl.3, 2012.

EL-LOLY, M. M. Composition, properties and nutritional aspects of milk fat globule membrane, a review. Food Nutr. Sci., v.61, p.7-32, 2011.

FAGNANI, R.; CARRARO, P.E.; BATTAGLINI, A.P.P.; ARAÚJO, J.P.A. Alterações na densidade e crioscopia do leite pela adição de diferentes concentrações de citrato e fortificante. Ver. Caatinga, v.27, p.208-215, 2014.

GENCUROVA, V.; HANUS, O.; VYLETELOVA, M et al. The relationships between goat and cow milk freezing point, milk composition and properties. Sci. Agric. Bohem. v.39, p.324-328, 2008.

HANUS, O.; HANUSOVÁ, K.; VYLETELOVÁ, M. et al. Selected abiotic factors that influence raw cow milk freezing point depression. Acta Vet. Brno. v.81, p.49$55,2012$.

HENNO, M.; OTS, M.; JÕUDU, I. et al. Factors affecting the freezing point stability of milk from individual cows. Int. Dairy J., v.18, p.210-215, 2008.

MATYSEK, M.K.; LITWINCZUK, Z.; FLOREK, M.; BARLOWSKA, J. The effects of breed and other factors on the composition and freezing point of cow's milk in Poland. Int. J. Dairy Techn., v.64, p.336-342, 2011.

MILK: determination of freezing point. Thermistor cryoscope method (reference method). Int. IDF Stand., n.108, p.24, 2009.
OFFICIAL methods of analysis of AOAC International. Method 947.05. 20.ed. Rockville: AOAC, 2016.

PINTIC-PUKEC, N.; BARAC, Z.; DAKIC, A. et al. Determination of the freezing point in cow milk samples preserved with azidiol. Mljekarstvo, v.61, p.336-340, 2011.

RODRIGUEZ-OTERO, J.L.; HERMIDA, M.; CENTENO, J. Analysis of dairy products by nearinfrared spectrocopy: a review. J. Agric. Food Chem., v.45, p.2815-2819, 1997.

RODRIGUEZ-SAONA, L.E.; KOCA, N.; HARPER, W.J. et al. Rapid determination of Swiss cheese composition by Fourier transform infrared/attenuated total reflectance spectroscopy. J. Dairy Sci., v.89, p.1407-1412, 2006.

SALA, C.; MORAR, A.; MORVAY, A. et al. Research regarding factors that influenced the variation of freezing milk. Lucr. Stiint. Med. Vet., v.43, p.204-211, 2010.

SAMPAIO, I.B.M. Estatística aplicada à experimentação animal. 4.ed. Belo Horizonte: Fundação de Estudo e Pesquisa em Medicina Veterinária e Zootecnia, 2015. 265p.

SÁNCHEZ, A.; SIERRA, D.; LUENGO, C. et al. Evaluation of the Milkoscan FT 6000 milk analyzer for determining the freezing pinto $\mathrm{f}$ goat's milk under diferente analytical conditions. J. Dairy Sci. v.90, p.3153-3161, 2007.

SENEVIRATHNE, P.G.N.D.; MANGALIKA, U.L.P.; ADIKARI, A.M.J.B.; NAYANANJALIE, W.A.D. Evaluation of cow factors and milk composition on freezing point depression of cow milk. Int. J. Liv. Res., v.6, p.61-67, 2016.

SHIPE, W.F. The freezing point of milk. A review. $J$. Dairy Sci., v.42, 1745-1762, 1959.

SILVEIRA, T.M.L.; FONSECA, L.M.; CANÇADO, S.V.; FERRAZ, V. Comparação entre métodos de referência e a análise eletrônica na determinação da composição do leite bovino. Arq. Bras. Med. Vet. Zootec., v.56, p.782-787, 2004.

STUART, B. Infrared spectroscopy: fundamentals and applications. West Sussex: John Wiley \& Sons, 2004. p.2-67.

ZAGORSKA, J.; CIPROVICA, I. Evaluation of factors affecting freezing point of milk. Int. J. Biol. Biomol. Agric. Food Biotechn. Engin., v.7, n.2, 2013. 